or due to a characteristic of the mechanism independent of this set of values.

After extrapolating $(d \sigma / d \Omega)_{\text {lab }}$ at larger angles, as indicated by the dashed line in Fig. 3, the integrated cross section for the production of quasi-Kr is about $500 \mathrm{mb}$. It is a large part of the reaction cross section (calculated value 870 $\mathrm{mb}$ ), and this seems to confirm the idea that the quasifission reactions occur instead of the complete-fusion reactions. One should remember, however, that the method used for the angular distribution is based on a hypothesis which is reasonable but not entirely proved for angles far away from $55^{\circ}$.

More work-with more intense beams if possible - has to be done in order to get more detailed information on the features of the quasifission reactions induced by $\mathrm{Kr}$ ions. Nevertheless we hope the indications we have obtained will stimulate theoretical work which is now underway for understanding the reaction mechanism. In particular, the angular distribution should help in deciding among the various pictures for the potential energy between the two nuclei which can be considered in order to explain the quasifission reactions. ${ }^{8}$

We thank N. Rowley for discussions and for reading the manuscript.

Note added. -We have recently made angulardistribution measurements at large angles. The maximum at $65^{\circ}$ has been confirmed but the descent above $80^{\circ}$ is not as steep as the dashed curve on Fig. 3: At $120^{\circ}$ the cross section has decreased down to $4 \mathrm{mb} / \mathrm{sr}$.

*Chercheur, Institut Interuniversitaire des Sciences Nucléaire, Université Libre de Bruxelles, Brussels, Belgium.

†Détaché de 1'Université de Clermont-Ferrand, Clermont-Ferrand, France.

${ }^{1} J$. Galin, D. Guerreau, M. Lefort, J. Péter, X. Tarrago, and R. Basile, Nucl. Phys. A159, 461 (1970).

${ }^{2}$ A. G. Artukh, G. F. Gridnev, V. L. Mikheev, V. V. Volkov, and J. Wilczynski, Joint Institute for Nuclear Research, Dubna, Report No. E7-6970, 1973 (to be published).

${ }^{3}$ L. G. Moretto, D. Heunemann, R. C. Jared, R. C. Gatti, and S. G. Thompson, International Atomic Energy Agency Symposium on Fission, Rochester, New York, 1973 (to be published), Paper No. SM174/75.

${ }^{4} \mathrm{~J}$. Péter, F. Hanappe, C. Ngô, and B. Tamain, Institut de Physique Nucléaire, Orsay, Report No. IPNO-RC-73-07, 1973 (to be published).

${ }^{5}$ B. Tamain, M. Lefort, C. Ngô, and J. Péter, J. Phys. (Paris) 33, Colloq. C5, Vol. 2, 50 (1972).

${ }^{6}$ A. J. Sierk and J. R. Nix, International Atomic Energy Agency Symposium on Fission, Rochester, New York, 1973 (to be published), Paper No. SM174/74.

${ }^{7}$ M. Lefort, C. Ngô, J. Péter, and B. Tamain, Nucl. Phys. A216, 166 (1973).

${ }^{8} \mathrm{~K}$. Dietrich, private communication.

\title{
Nuclear Shock Waves in Heavy-Ion Collisions
}

\author{
Werner Scheid, Hans Müller, and Walter Greiner \\ Institut fiir Theoretische Physik der Universität Frankfurt, Frankfurt am Main, Germany
} (Received 19 November 1973)

\begin{abstract}
It is shown that nuclear matter is compressed during the encounter of heavy ions. If the relative velocity of the nuclei is larger than the velocity of first sound in nuclear matter (compression sound for isospin $T=0$ ), nuclear shock waves occur. They lead to densities which are 3-5 times higher than the nuclear equilibrium density $\rho_{0}$, depending on the energy of the nuclei. The implications of this phenomenon are discussed.
\end{abstract}

The possibility of compression of nuclear matter in nucleus-nucleus collisions in one of the most interesting aspects of heavy-ion physics. It has been discussed earlier in connection with the sudden nucleus-nucleus potentials ${ }^{1}$ and their energy dependence, ${ }^{2}$ which seems to confirm the experimentally deduced heavy-ion potentials of the Yale group. ${ }^{3}$ These earlier considerations are valid as long as the relative heavy-ion velocity $v_{r}$ does not exceed the velocity of first sound in nuclear matter $c_{s}$; i.e., for $v_{r}<c_{s}$. Here the first sound is an isospin $T=0$ compression wave while the second sound describes an isospin $T=1$ wave where a proton-neutron separation travels in constant nuclear matter density $\rho_{0}=\rho_{p}+\rho_{n}{ }^{4}$

In order to study local compression effects for 
the cases $v_{r}>c_{s}$ in head-on nucleus-nucleus collisions, we propose the following very simplified model: We restrict ourselves to two identical nuclei whose volume is divided into three parts [see Fig. 1(a)], namely an ellipsoid with axes $a$ and $b$ sandwiched between two cut-off spheres with radius $R$ and relative distance $r$. The system will clearly be rotationally symmetric around the $z$ axis, connecting the centers of the two spheres and of the ellipsoid. The nuclear matter is assumed to be homogeneously distributed over the different volumes. Compression $\left(\rho>\rho_{0}\right)$ should only occur in the ellipsoidal region. The four coordinates $a, b, r$, and $R$ define the geometry of the system. We choose, however, $R=$ const in the collision so that we are dealing with three degrees of freedom only. This approximation is rather inessential for the results, but simplifies the theoretical considerations considerably.

If the density in the cut-off spheres is the equilibrium density $\rho_{0}$, the density in the ellipsoidal region follows from matter conservation as

$$
\begin{aligned}
\rho_{1}=\rho_{0}\left[\left(R^{3} / a b^{2}\right)(1+\alpha)^{2}\right. & \left(1-\frac{1}{2} \alpha\right) \\
& \left.+(1-\beta)^{2}\left(1+\frac{1}{2} \beta\right)\right],
\end{aligned}
$$

where $\alpha=(s-\gamma) / 2 R$ and $\beta=s / 2 a$, with $s$ the distance between the intersection points of the two spheres with the ellipsoid [see Fig. 1(a)]. The various types of shapes shown in Fig. 1(b) are possible, depending on the relative size of the ellipsoid and the spheres; they can be characterized by the quantity

$$
\delta=b^{2}-R^{2}+\left(\frac{1}{2} r\right)^{2} .
$$

We will later exclude the case $\delta<0$, because it corresponds to an ellipsoid with compressed matter embedded completely within the two spheres, which is unphysical. The distance $s$ is given by

$$
s=\frac{r}{\epsilon}\left[\left(1+\frac{4 \epsilon \delta}{r^{2}}\right)^{1 / 2}-1\right]
$$

for $\delta>0$, where $\epsilon=b^{2} / a^{2}-1$, and $s \equiv 0$ for $\delta<0$.

Because the solution of the full hydrodynamical problem is too complicated, we make the following approximations for the calculation of the $\mathrm{ki}$ netic energy $E=\frac{1}{2} M \int \rho^{3} v^{2} d \tau$ : (I) The velocity field of the matter in the cut-off spheres is homogeneous and equal to the velocity of the respective centers, i.e.,

$$
\overrightarrow{\mathrm{v}}= \pm \frac{1}{2} \vec{r}_{\mathbf{e}} \text {. }
$$

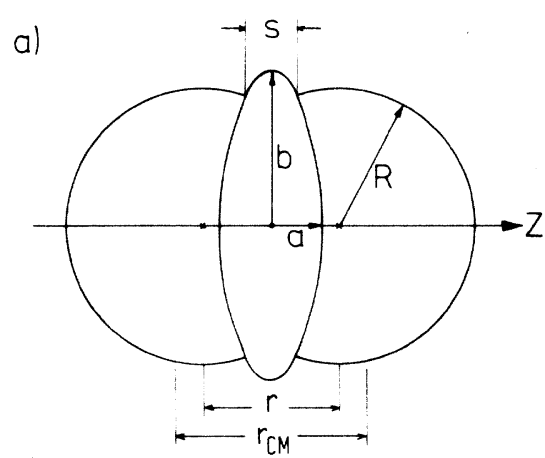

b)

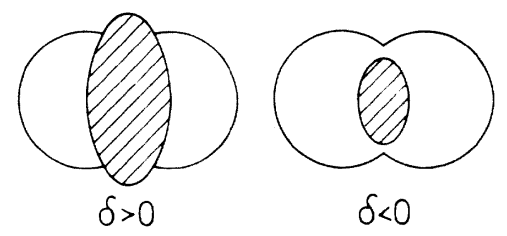

FIG. 1. (a) Geometric parameters of the model. (b) Two cases $\delta>0$ and $\delta<0$. The unphysical situation $\delta<0$ is excluded by forces of constraints.

(II) The velocity field in the ellipsoid is irrotational and can thus be derived from a potential through the equations

$$
\begin{aligned}
& \overrightarrow{\mathrm{v}}=\operatorname{grad} \varphi, \\
& \Delta \varphi=\rho_{1}^{-1} \partial \rho_{1} / \partial t .
\end{aligned}
$$

The boundary condition $v=\dot{b}$ and $\overrightarrow{\mathrm{v}} \cdot \overrightarrow{\mathrm{e}}_{z}=0$ along the circle $z=0, x^{2}+y^{2}=b^{2}$ gives for the solution of (4)

$$
\overrightarrow{\mathbf{v}}=\frac{\dot{b}}{b}\left(x \overrightarrow{\mathbf{e}}_{x}+y \overrightarrow{\mathbf{e}}_{y}\right)-\left(\frac{1}{\rho_{1}} \frac{\partial \rho_{1}}{\partial t}+\frac{2 \dot{b}}{b}\right) z \overrightarrow{\mathbf{e}}_{z},
$$

which allows with (3) the calculation of the kinetic energy in the form

$$
E_{\mathrm{kin}}=\frac{1}{2} \sum_{i, k} m_{i k} \dot{q}_{i} \dot{q}_{k}
$$

where $q_{i}=\{r, a, b\}$.

If the Coulomb energy is disregarded, the potential energy is given by the compression energy $^{4}$ and the heat energy of the Fermi gas ${ }^{5}$ in the ellipsodial region, ${ }^{6}$ i.e.,

$$
\begin{aligned}
E_{\text {pot }} & =\int W \rho d \tau=W\left(\rho_{1}\right) \rho_{1} \frac{4}{3} \pi a b^{2} \\
& =\left(C / 2 \rho_{0}\right)\left(\rho_{1}-\rho_{0}\right)^{2} \times \frac{4}{3} \pi a b^{2},
\end{aligned}
$$

with

$$
W(\rho)=\left(C / 2 \rho_{\rho} \rho\right)\left(\rho-\rho_{0}\right)^{2}+\frac{1}{2} \beta T^{2} \rho^{-2 / 3},
$$

$T$ being the temperature in the compressed region. To avoid unphysical shapes with $\delta<0$, a 
potential of constraints $\frac{1}{2} V_{0} \delta^{2}$ is added for $\delta<0$ to the potential energy. It forces the system to shapes with $\delta>0$. The strength $V_{0}$ has negligible influence on the results.

Since we have introduced regions of different densities $\rho_{1}$ and $\rho_{0}$, the following boundary conditions along the surface between $\rho_{1}$ and $\rho_{2}$ have to hold ${ }^{7}$ :

$$
\begin{aligned}
& {[\rho U]=0, \quad[\rho U \overrightarrow{\mathrm{v}}+p \overrightarrow{\mathrm{n}}]=0,} \\
& {\left[\rho U\left(1 / 2 \overrightarrow{\mathrm{v}}^{2}+w\right)+p \overrightarrow{\mathrm{v}} \cdot \overrightarrow{\mathrm{n}}\right]=0,}
\end{aligned}
$$

The brackets $[f]$ denote the jump of the quantity $f$ across the surface, i.e., $[f] \equiv f_{1}-f_{2} ; \overrightarrow{\mathbf{n}}$ is the unit vector on the surface, and $U=\overrightarrow{\mathrm{v}} \cdot \overrightarrow{\mathrm{n}}-G$, where $G$ is the speed of advance of the surface. The pressure $p$ is related to the density of the potential energy according to (7):

$$
p=\rho^{2} \frac{d w}{d \rho} \underset{\text { constentropy }}{=} \frac{C}{2 \rho_{0}}\left(\rho^{2}-\rho_{0}^{2}\right)+\frac{\beta}{3} T^{2} \rho^{1 / 3} .
$$

From Eqs. (8) it follows that

$$
\beta T^{2} \rho^{1 / 3}=\frac{3}{8} \frac{C}{\rho_{0}}\left(\rho_{1}-\rho_{0}\right)^{2} \frac{\rho_{1} / \rho_{0}-1}{1-\rho_{1} / 4 \rho_{0}} .
$$

Restricting ourselves, for simplicity, to the case that $\rho$ does not deviate too much from $\rho_{0}$, we disregard the temperature dependence. Furthermore, because the velocity field is already given as a function of the coordinates $a, b$, and $r$, the above Eqs. (8) connect the velocities $\dot{a}, \dot{b}$, and $\dot{r}$. For simplicity we only fulfill these equations at the points $z= \pm a, x=y=0$ and obtain

$$
\dot{a}=c_{s}\left[\frac{1}{2} \frac{\rho_{1}}{\rho_{0}}\left(1+\frac{\rho_{1}}{\rho_{0}}\right)\right]^{1 / 2}+\frac{\dot{r}}{2}
$$

where $c_{s}=(C / M)^{1 / 2}$ is the velocity of first sound, with $M$ the nucleon mass. This indicates that the coordinate $a$ is not an independent degree of freedom in this model.

The total energy of the system,

$$
\boldsymbol{E}=\frac{1}{2} \sum_{i, k} m_{i k} \dot{q}_{i} \dot{q}_{k}+E_{\text {pot }},
$$

has to be constant in time, i.e., $d E / d t=0$. The solutions of (11) have to fulfill the additional condition (10), which serves as an equation of motion. Introducing the momenta $\hbar_{k}=\sum_{i} m_{k i} \dot{q}_{i}$, the three equations of motion to be solved are

$$
\begin{aligned}
& \partial E / \sigma q_{3}+\dot{p}_{3}=0 \\
& \left(\partial E / \partial q_{1}+\dot{p}_{1}\right) \dot{q}_{1}+\left(\partial E / \partial q_{2}+\dot{p}_{2}\right) \dot{q}_{2}=0 \\
& d \psi / d t=0
\end{aligned}
$$

with

$$
\psi=\frac{\dot{q}_{1}}{2}-\dot{q}_{2}+c_{s}\left[\frac{1}{2} \frac{\rho_{1}}{\rho_{0}}\left(1+\frac{\rho_{1}}{\rho_{0}}\right)\right]^{1 / 2}=0 .
$$

The calculations are carried out for ${ }^{16} \mathrm{O}-{ }^{16} \mathrm{O}$ scattering. The two parameters of the model are chosen as $C=\frac{100}{9} \mathrm{MeV}$, which corresponds to a compressibility $K=100 \mathrm{MeV}$, and $\rho_{0}=0.138 \mathrm{fm}^{-3}$ (see Ref. 2). The initial conditions for $t=0$ are $r=2(R-\Delta R)$ with $\Delta R=\frac{1}{20} R, \rho_{1}=2 \rho_{0}, l=(2 R \Delta R)^{1 / 2}$, $\dot{b}=-\frac{1}{2}(R / l) \dot{r}$. The initial compression $\rho_{1}=2 \rho_{0}$ in the surface zone does not influence the results, as can be seen from $\rho_{1}(t)$ in Fig. 2. The initial conditions for all other coordinates $a, \dot{r}$, and $\dot{a}$ are determined by Eqs. (1), (10), and (11).

Figure 2 shows the results for the time dependence of $r, a, l, r_{\text {c.m. }}$, and $\rho_{1}$. Here $r_{\text {c.m. }}$. means the distance between centers of mass of the two halves of the nuclear system [see Fig. 1 (a) ]. Clearly, the model can only be used as long as $\frac{1}{2} r+R>a$ is fulfilled. Obviously the radi-

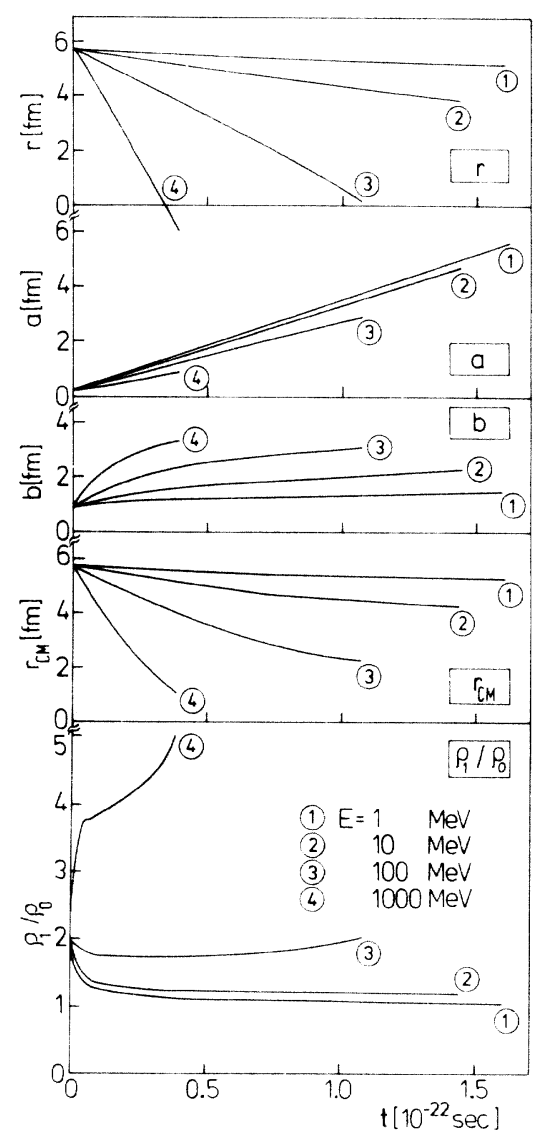

FIG. 2. Parameters $\boldsymbol{r}, \boldsymbol{a}, \boldsymbol{b}, \boldsymbol{r}_{\mathrm{c} . \mathrm{m} .}$, and $\rho_{1} / \rho_{0}$ as a function of time for energies $E_{\mathrm{c} . \mathrm{m} .}=1,10,100$, and $1000 \mathrm{MeV}$. 


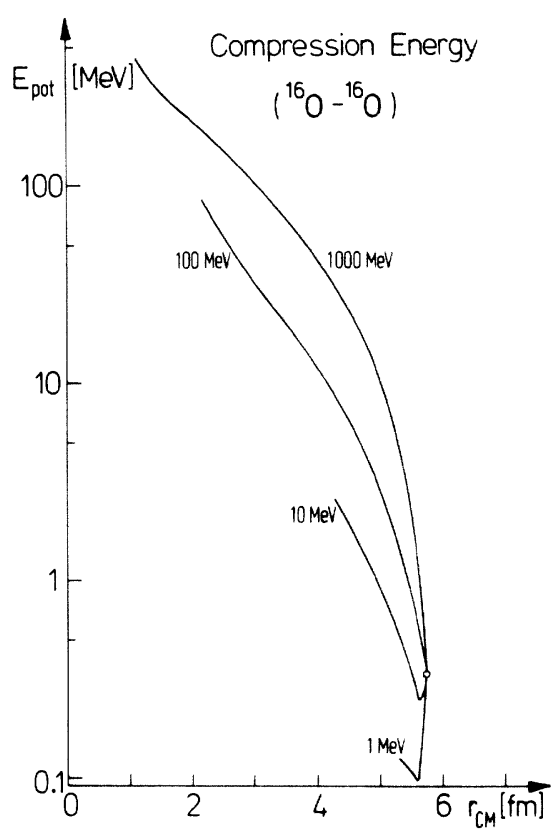

FIG. 3. Potential energy as a function of the centerof-mass distance. It is strongly energy-dependent and shows a strong soft core, particularly for high energles.

us $a(t)$ increases with nearly constant velocity, in contrast to $b(t)$ which increases faster for higher energies. This means that matter is pushed outwards perpendicular to the relative motion of the two nuclei. Note that for small energies the density $\rho_{1}$ is first decreasing rapidly and then again increasing slowly at larger times. The fast decrease in the beginning is the adjustment of the compressed nuclear density from the initial condition $\rho_{1}=2 \rho_{0}$ to the final density. Therefore the results are independent of the initial conditions.

Already for a bombarding energy of $\boldsymbol{E}_{\text {c.m. }}=100$ $\mathrm{MeV}$, which corresponds to a laboratory energy of $E_{\mathrm{lab}} / A=12.5 \mathrm{MeV} /$ nucleon of the ${ }^{16} \mathrm{O}$ projectile, a density compression of $\rho_{1} / \kappa_{0}=2$ results. This indicates a nuclear matter shock wave, which becomes even more pronounced $\left(\rho_{1} \approx 5 \rho_{0}\right)$ for very high nucleus-nucleus energies $(\geqslant 100 \mathrm{MeV} / \mathrm{nu}-$ cleon). In this latter case it is necessary to investigate the relativistic hydrodynamics. Figure 3 shows the potential energy, which is stored in the compressed matter of the ellipsoid, as a function of $r_{\text {c.m. }}$. It obviously leads to a strong energy-dependent soft-core potential. The soft core reaches the magnitude of a few $\mathrm{MeV}$ for $\mathrm{O}^{16}$ ions of $10 \mathrm{MeV}$ to several hundred $\mathrm{MeV}$ for $\mathrm{O}^{16}$ ions of $1000 \mathrm{MeV}$.

The strongly compressed nuclear system shows up experimentally in the strong energy-dependent soft-core potential for nucleus-nucleus scattering. It should be observable in the backward direction of elastic scattering. This elastic cross section is expected to be very small because of the large number of inelastic channels available as a result of the highly compressed matter. The systematic investigation of the elastic and inelastic excitation functions in nucleus-nucleus collision should, however, give information on the nuclear compressibility. The time dependence of $a(t)$ and $b(t)$ (Fig. 2) indicates that the shock wave is expanding faster along the beam direction $[a(t)$ grows faster than $b(t)]$. Therefore the fragments are expected to peak forward and backward in the c.m. system, i.e., forward in the lab system.

It should be realized that the results are valid for all nuclei and not only for ${ }^{16} \mathrm{O}-{ }^{16} \mathrm{O}$ collisions, i.e., in particular also for $\mathrm{Pb}-\mathrm{Pb}$ or $\mathrm{U}-\mathrm{U}$ collisions at high energies. In these latter cases one can also expect strong nuclear shock waves with $\rho_{1} \approx(3-5) \rho_{0}$. If matter is compressed to such an extent, these systems can be overcritical for the pionic field in analogy to similar phenomena for the electron-positron fields in superheavy intermediate molecules ${ }^{8}$ : Because of the pion-nucleus interaction the binding energy of bound pion-atomic states can become larger than $2 m_{\pi} c^{2}$ ("diving of pionic bound states"), and $\pi^{+}-\pi^{-}$pairs or $\pi^{0}$ pairs may be produced without cost of energy. Since pions are bosons, such pairs can be created until either a repulsive pion-pion interaction $\left(\varphi^{4}\right.$ term) or a "pionic Pauli principle" can prevent further production. A "pionic Pauli principle" can occur, for example, if the pions are para-particles; i.e., they obey, for example, para-Fermi statistics, which would stabilize the pion vacuum. Depending on the degree of such a para-statistics, the energy in the nuclear matter of the highly compressed overcritical system will condense into pionic matter. It is not yet clear whether such mixed pionic-nuclear matter can be stable as, e.g., a pionic drop (new vacuum state for hadronic matter) or whether it will dissipate into many pions (multiple pion production, pionic showers). In any case, these new "degrees of freedom" for overcritical nuclear matter seem to give not only a clear indication for the intermediate highly compressed nuclear matter, but also new, exciting prospects for highenergy heavy-ion physics, in that fundamental 
properties of hadronic matter can eventually be tested.

${ }^{*}$ Work supported by the Bundeministerium für Forschung und Technologie and by the Gesellschaft für Schwerionenforschung (GSI).

${ }^{1} \mathrm{~K}$. Pruess and W. Greiner, Phys. Lett. 33B, 197 (1970); W. Greiner and W. Scheid, Suppl. J. Phys. (Paris) 32, C6-91 (1971).

${ }^{2}$ H. Müller, W. Scheid, and W. Greiner, to be published.

${ }^{3} \mathrm{D}$. A. Bromley, in Proceedings of the International Conference on Nuclear Reactions Induced by Heavy Ions, Heidelberg, Germany, 1969, edited by R. Bock and W. R. Herring (North-Holland, Amsterdam, 1970), p. 27 .
${ }^{4}$ Nuclear hydrodynamics has been discussed and justified by M. Danos, University of Maryland Technical Report No. 221, 1961 (unpublished); W. Wild, Sitzungsber. Math.-Naturwiss. K1. Bayer. Akad. Wiss. München $\underline{1955}$, 371; A. E. Glassgold, W. Heckroth, and K. M. Watson, Ann. Phys. (Paris) 6 , 1 (1959).

${ }^{5}$ L. D. Landau and E. M. Lifshitz, Statistical Physics (Pergamon, London, 1958).

${ }^{6}$ J. D. Walecka, Phys. Rev. 126, 653 (1962); K. Woeste, Z. Phys. 133, 370 (1952); H. A. Bethe, Annu. Rev. Nucl. Sci. 21, 93 (1971).

${ }^{7} \mathrm{~J}$. Serrin, in Handbuch der Physik, edited by S. Flügge (Springer, Berlin, 1959), Vol. 8 Part I, p. 125 .

${ }^{8}$ B. Müller, H. Peitz, J. Rafelski, and W. Greiner, Phys. Rev. Lett. 28, 1235 (1972); B. Müller, J. Refelski, and W. Greiner, Z. Phys. 257, 62, 183 (1972).

\section{ERRATUM}

MEASUREMENTS OF ELECTRON DENSITY EVOLUTION AND BEAM SELF-FOCUSING IN A LASER-PRODUCED PLASMA. L. C. Johnson and T. K. Chu [Phys. Rev. Lett. 32, 517 (1974)].

Reference 1 is incor rect. It should read as follows:

${ }^{1}$ J. M. Dawson, R. E. Kidder, and A. Hertzberg, Princeton University Plasma Physics Laboratory, MATT Report No. 782, 1971 (unpublished); J. M. Dawson, A. Hertzberg, R. Kidder, G. Vlases, H. Ahlstron, and L. Steinhauer, in Proceedings of the Fourth Conference on Plasma Physics and Controlled Nuclear Fusion, Madison, Wisconsin, 1971 (International Atomic Energy Agency, Vienna, Austria, 1972), Vol. I, p. 673 . 